\title{
Visualizing the phonon wave function
}

\author{
Scott C. Johnson and Thomas D. Gutierrez
}

\begin{abstract}
A phonon often is described as "a quantum of lattice vibration," but this description can be difficult to reconcile with the wave functions explored in a typical undergraduate quantum mechanics class. A phonon wave function is similar to the harmonic oscillator wave functions studied in introductory quantum mechanics, except that it is many-dimensional. We suggest a way to visualize the probability density for this very high-dimensional wave function. The resulting pictures are especially clear and intuitive for a coherent state, which is both a good approximation to a sound wave and a discrete analog to laser light. These pictures can also provide a qualitative introduction to quantum field theory.
\end{abstract}

\section{INTRODUCTION}

Phonons and quantum field theory are usually regarded as graduate topics but undergraduate students often are curious about them. The goal of this article is to give a semiquantitative introduction to these topics at a level appropriate for the final weeks of an introductory quantum mechanics class. At this level, students are comfortable with onedimensional wave functions and may have been introduced to higher dimensional wave functions. This article takes this background into account and presents a phonon or a quantum field as simply another wave function, albeit a wave function of many coordinates instead of the usual one, two, or three. It is challenging to visualize a function in more than three dimensions, but we claim that some intuition can be gained by looking at groups of one- and two-dimensional projections of such functions. A related series of pictures can be generated by the software in Ref. 1 .

Understanding Fig. 18 is the key to our visualization technique. It shows a propagating coherent state of the phonon field. Most of this article up to this point describes the various concepts and techniques used to generate this figure. First, the analysis of a two-particle coupled harmonic oscillator system is given using our visualization scheme, both classically and quantum mechanically. This system is considered to establish our notation and to illustrate the idea of using multiple projections of a function to visualize it. Then, an eight-particle lattice is analyzed in detail, emphasizing some of the very interesting states of this system and culminating in Fig. 18. Some other interesting states of the eightoscillator system are then discussed and a comparison is made to a quantum field.

\section{A TWO-PARTICLE SYSTEM}

A system of two coupled harmonic oscillators makes an excellent system for demonstrating many phonon concepts because this system can be thought of as a very small (twoparticle) lattice. In particular, it demonstrates normal mode decomposition and how the wave functions are separable, and hence much simpler, when expressed in normal mode coordinates. Best of all, the probability density of the entire wave function in position space can be fully visualized, so that we can develop some intuition about projections onto the one-dimensional position or normal mode axes. An excellent treatment of this system is given in Shankar. ${ }^{2}$
We first do a classical analysis. The system is shown in Fig. 1. Each mass is constrained to move vertically, and we consider only small oscillations so that this system is a coupled pair of one-dimensional (1D) harmonic oscillators. Vertical oscillations are chosen because they correspond directly to the axes of our plots. The position coordinates of the masses are $q_{1}$ and $q_{2}$. The momenta, which are directed vertically along each coordinate, are denoted as $p_{1}$ and $p_{2}$, respectively. The masses of the two particles are assumed to be identical, $m$, and the spring constants are also identical, $\kappa$.

The key to analyzing this system and to understanding its behavior is to use normal mode coordinates, denoted by the upper-case letters $Q_{1}$ and $Q_{2}$. For this system, they are

$$
\begin{aligned}
& Q_{1}=\frac{1}{\sqrt{2}}\left(q_{2}+q_{1}\right), \\
& Q_{2}=\frac{1}{\sqrt{2}}\left(q_{2}-q_{1}\right) .
\end{aligned}
$$

The momentum coordinates conjugate to $Q_{1}$ and $Q_{2}$ are $P_{1}$ and $P_{2}$, and are related to the momenta of each mass by a similar set of equations,

$$
\begin{aligned}
& P_{1}=\frac{1}{\sqrt{2}}\left(p_{2}+p_{1}\right), \\
& P_{2}=\frac{1}{\sqrt{2}}\left(p_{2}-p_{1}\right) .
\end{aligned}
$$

In position coordinates the equations of motion are coupled as indicated by a nondiagonal force matrix in the equation of motion,

$$
\left[\begin{array}{cc}
2 \kappa / m & -\kappa / m \\
-\kappa / m & 2 \kappa / m
\end{array}\right]\left[\begin{array}{l}
q_{1} \\
q_{2}
\end{array}\right]+m\left[\begin{array}{l}
\ddot{q}_{1} \\
\ddot{q}_{2}
\end{array}\right]=0 .
$$

In normal mode coordinates, however, the equations of motion are not coupled as indicated by the diagonal force matrix,

$$
\left[\begin{array}{cc}
\kappa / m & 0 \\
0 & 3 \kappa / m
\end{array}\right]\left[\begin{array}{l}
Q_{1} \\
Q_{2}
\end{array}\right]+m\left[\begin{array}{l}
\ddot{Q}_{1} \\
\ddot{Q}_{2}
\end{array}\right]=0 .
$$

This is the reason for introducing and using normal mode coordinates - each of the normal modes can be treated as if it 


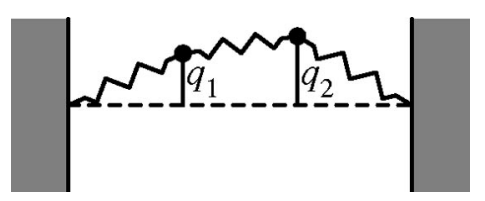

Fig. 1. A two-particle system, showing the position coordinates $q_{1}$ and $q_{2}$ of the two masses (dots), which are constrained to move vertically, connected by springs.

were an independent, one-dimensional harmonic oscillator.

Figure 2 shows three ways of plotting the configuration of this system at any time. The pair of one-dimensional $q_{1}$ and $q_{2}$ axes on the left simply gives the location of each of the masses. (The horizontal line crossing both axes indicates the origin or equilibrium position.) The two-dimensional plot in the center specifies the location of both masses with a single point. The pair of plots on the right gives the projection onto each of the normal mode axes. These three views will be used consistently to visualize the two-oscillator system in this section.

It is helpful to look at a few examples of configurations plotted on these axes, as given in Fig. 3. In Fig. 3(a), for example, the dot lies along the $Q_{1}$ axis and the system is in one of its two normal modes. The projections onto $q_{1}$ and $q_{2}$ show that in this normal mode, both masses are displaced identically. The projections onto $Q_{1}$ and $Q_{2}$ show that the system is only in normal mode 1 . In this mode, the dot on the two-dimensional (2D) plot will oscillate only along the $Q_{1}$ axis about the origin and the masses will oscillate in unison about the equilibrium positions. Figure 3(b) shows the other normal mode, where the masses oscillate against each other. Figure 3(c) shows only mass 2 being displaced. The time evolution from this initial displacement will not be a simple oscillation like a normal mode, but a more complex motion that is a superposition of the two normal modes.

We next analyze this system quantum mechanically. We seek state vectors $|\psi(t)\rangle$ that satisfy the Schrödinger equation

$$
i \hbar \frac{d}{d t}|\psi(t)\rangle=\hat{H}|\psi(t)\rangle,
$$

where $\hat{H}$ is the Hamiltonian operator. In position coordinates, the Hamiltonian is

$$
\hat{H}=\frac{\hat{p}_{1}^{2}}{2 m}+\frac{\hat{p}_{2}^{2}}{2 m}+\frac{1}{2} m \omega^{2}\left[\hat{q}_{1}^{2}+\hat{q}_{2}^{2}+\left(\hat{q}_{1}-\hat{q}_{2}\right)^{2}\right],
$$
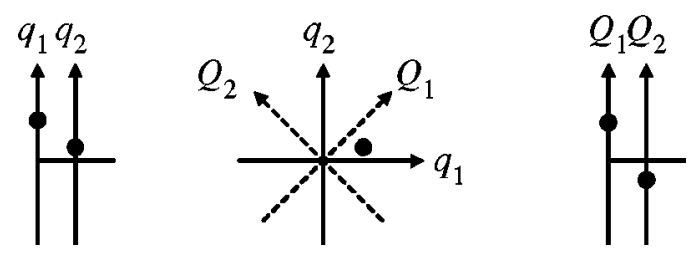

Fig. 2. Three views of the two-dimensional space formed by $q_{1}$ and $q_{2}$. The center view shows the single point in this space which gives the locations of both masses. The position axes $q_{1}$ and $q_{2}$ are shown as solid lines and the normal mode axes $Q_{1}$ and $Q_{2}$ are shown as dashed lines. The left view shows its projection onto the $q_{1}$ and $q_{2}$ axes, and the right view onto the normal mode axes $Q_{1}$ and $Q_{2}$. (a)
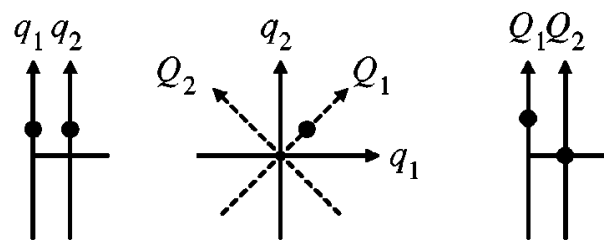

(b)
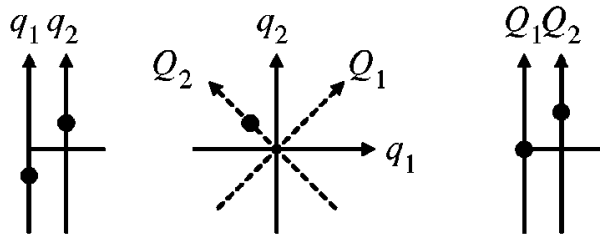

(c)
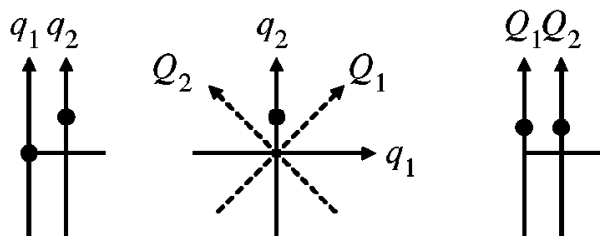

Fig. 3. Plot of the system in each of its two eigenmodes (a) and (b). The amplitudes of the displacements are arbitrary. (c) The system with one mass displaced but the other at its equilibrium position.

where $\omega^{2}=\kappa / m$. To make the transition from the classical to the quantum mechanical analysis of the same system, the position and momentum coordinates are changed to operators as indicated by the caret. For example, $\hat{q}_{1}$ is the operator corresponding to the coordinate $q_{1}$.

The Hamiltonian, as written in position coordinates in Eq. (6), does not give a separable Schrödinger wave equation because of the $\left(\hat{q}_{1}-\hat{q}_{2}\right)^{2}$ term. However, in normal mode coordinates,

$$
\hat{H}=\frac{\hat{P}_{1}^{2}}{2 m}+\frac{\hat{P}_{2}^{2}}{2 m}+\frac{1}{2} m \omega_{1}^{2} \hat{Q}_{1}^{2}+\frac{1}{2} m \omega_{2}^{2} \hat{Q}_{2}^{2},
$$

it does give a separable wave equation. Here, $\omega_{1}^{2}=\kappa / m$ and $\omega_{2}^{2}=3 \kappa / m$ are the eigenvalues for normal mode coordinates $Q_{1}$ and $Q_{2}$, which are the eigenmodes of the system.

Because the wave equation separates in normal mode coordinates, these coordinates are the easiest to use. Solutions to the Schrödinger equation for this system can be written as simple products of the familiar one-dimensional harmonic oscillator states. For example, the ground state of the system is the product of ground state wave functions for $Q_{1}$ and for $Q_{2}$,

$$
\begin{aligned}
\psi\left(Q_{1}, Q_{2}\right)= & \psi_{0}\left(Q_{1}\right) \psi_{0}\left(Q_{2}\right) \\
= & \left(\frac{m \omega_{1}}{\pi \hbar}\right)^{1 / 4} \exp \left(-\frac{m \omega_{1} Q_{1}^{2}}{2 \hbar}\right) \\
& \times\left(\frac{m \omega_{2}}{\pi \hbar}\right)^{1 / 4} \exp \left(-\frac{m \omega_{2} Q_{2}^{2}}{2 \hbar}\right) \\
= & \langle Q \mid 0,0\rangle .
\end{aligned}
$$

The last line uses Dirac notation. The state vector, or ket, is written as $|0,0\rangle$, which is of the form $\left|n_{1}, n_{2}\right\rangle$, where $n_{1}$ and $n_{2}$ indicate the energy eigenstate of the wave function along $Q_{1}$ and $Q_{2}$ respectively. Hence, $|0,0\rangle$ means that $n_{1}=0$ and $n_{2}=0$ and the system is in its ground state along both normal 
axes. This state vector exists in an infinite-dimensional Hilbert space and can be projected onto any set of basis vectors that span the space, such as an energy basis, a coordinate basis, or a momentum basis. One such set of coordinate basis vectors corresponds to the infinite set of points on the $Q_{1}-Q_{2}$ plane and is represented by the shorthand notation $\langle Q|$. The Hilbert space projection $\langle Q \mid 0,0\rangle$ gives the twodimensional wave function $\psi\left(Q_{1}, Q_{2}\right)$. Note that all the other projections in this section are done in this $2 \mathrm{D}$ coordinate space, not in the Hilbert space.

Note that the one-dimensional harmonic oscillator states do not lie along the position coordinates $q_{1}$ and $q_{2}$, but along the normal mode coordinates $Q_{1}$ and $Q_{2}$. Thus, it is easy to project the two-dimensional total probability density onto the one-dimensional $Q_{1}$ and $Q_{2}$ axes,

$$
P\left(Q_{1}\right)=\left|\psi_{0}\left(Q_{1}\right)\right|^{2} \text {. }
$$

It is not so easy, however, to project it onto the $q$ axes,

$$
P\left(q_{1}\right)=\int_{-\infty}^{\infty}\left|\psi\left(Q_{1}, Q_{2}\right)\right|^{2} d q_{2} .
$$

Typically, this integration must be done numerically. For the plots in this section, the numerical integration is done using the standard trapezoid method. For the plots in Sec. III, it is done using a Monte Carlo method.

As for any one-dimensional harmonic oscillator, we can define raising and lowering operators (also called creation and annihilation operators). Each normal mode coordinate has one raising and one lowering operator,

$$
\begin{aligned}
& \hat{A}_{1}^{\dagger}=\sqrt{\frac{m \omega_{1}}{2 \hbar}} \hat{Q}_{1}-i \sqrt{\frac{1}{2 m \omega_{1} \hbar}} \hat{P}_{1}, \\
& \hat{A}_{1}=\sqrt{\frac{m \omega_{1}}{2 \hbar}} \hat{Q}_{1}+i \sqrt{\frac{1}{2 m \omega_{1} \hbar}} \hat{P}_{1}, \\
& \hat{A}_{2}^{\dagger}=\sqrt{\frac{m \omega_{2}}{2 \hbar}} \hat{Q}_{2}-i \sqrt{\frac{1}{2 m \omega_{2} \hbar}} \hat{P}_{2}, \\
& \hat{A}_{2}=\sqrt{\frac{m \omega_{2}}{2 \hbar}} \hat{Q}_{2}+i \sqrt{\frac{1}{2 m \omega_{2} \hbar}} \hat{P}_{2} .
\end{aligned}
$$

The action of these operators on a ket is to raise or lower one of the $n$ values, the energy eigenstate of the corresponding mode,

$$
\begin{aligned}
& \hat{A}_{1}^{\dagger}\left|n_{1}, n_{2}\right\rangle=\sqrt{n_{1}+1}\left|n_{1}+1, n_{2}\right\rangle, \\
& \hat{A}_{1}\left|n_{1}, n_{2}\right\rangle=\sqrt{n_{1}}\left|n_{1}-1, n_{2}\right\rangle, \\
& \hat{A}_{2}^{\dagger}\left|n_{1}, n_{2}\right\rangle=\sqrt{n_{2}+1}\left|n_{1}, n_{2}+1\right\rangle, \\
& \hat{A}_{2}\left|n_{1}, n_{2}\right\rangle=\sqrt{n_{2}}\left|n_{1}, n_{2}-1\right\rangle .
\end{aligned}
$$

A few examples are $\hat{A}_{1}^{\dagger}|0,0\rangle=|1,0\rangle, \hat{A}_{1}^{\dagger} \hat{A}_{1}^{\dagger}|0,0\rangle=\sqrt{2}|2,0\rangle$, $\left(\hat{A}_{1}^{\dagger}\right)^{3}|0,0\rangle=\sqrt{6}|3,0\rangle, \quad \hat{A}_{1}|4,1\rangle=2|3,1\rangle, \quad \hat{A}_{2}^{\dagger}|2,8\rangle=3|2,9\rangle$, and $\hat{A}_{2}|3,0\rangle=0$.

The quantum mechanical version of this system can be visualized using the same sets of coordinates as the classical version. Instead of plotting a single point on each plot, the probability density is plotted at each point using a gray scale. Figure 4 shows four examples. Figure 4(a) shows the ground state for the two-particle system as a whole, given explicitly by Eq. (8). Each of the other plots is an excited state. For (a)
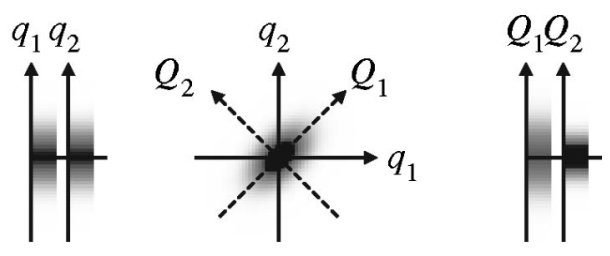

(b)
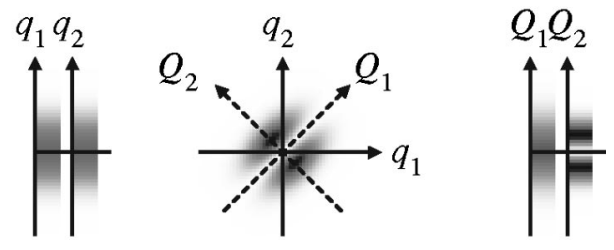

(c)
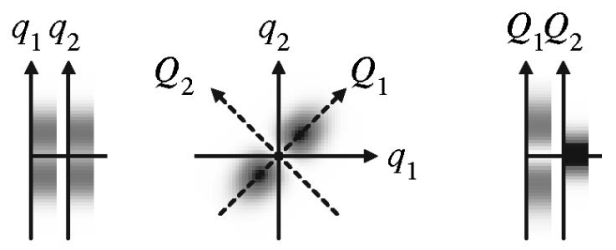

(d)
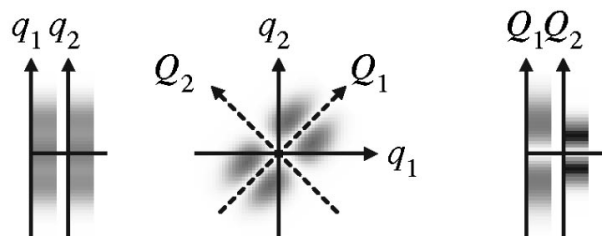

Fig. 4. Probability densities of four states of the two-particle system, (a) $|0,0\rangle$, (b) $|0,1\rangle$, (c) $|1,0\rangle$, and (d) $|1,1\rangle$.

each state, the center, two-dimensional plot is the most complete representation of the probability density function. It has the disadvantage, however, that such a plot cannot be made for functions of higher than three dimensions. The other sets of one-dimensional plots do not give as much information and are probably not as easy to interpret, but they can be easily adapted to visualizing higher-dimensional functions. This feature of the one-dimensional projections is the reason we use them to visualize the many-dimensional phonon wave function considered in Sec. III.

The projections onto the position axes (the left-hand plots) give the probabilities of finding masses 1 and 2 at various locations along the position coordinates $q_{1}$ and $q_{2}$. The horizontal width of these plots has no physical meaning and is chosen to make the probability densities easy to see. The projections onto the normal mode axes $Q_{1}$ and $Q_{2}$ (the righthand plots) are similar except they usually lack the intuitive explanation of the $q_{1}$ and $q_{2}$ projections. For this system, the $Q_{1}$ projection gives the probability density for the center of mass of the system because $Q_{1}=1 / \sqrt{2}\left(q_{1}+q_{2}\right)$; the $Q_{2}$ projection gives the probability density for the relative coordinate $1 / \sqrt{2}\left(q_{1}-q_{2}\right)$. In these plots, it is clear that the complete wave function is a product of functions along the dashed $Q_{1}$ and $Q_{2}$ axes, not the solid $q_{1}$ and $q_{2}$ axes.

All the states shown in Fig. 4 are eigenfunctions of this system, so they do not vary with time. The superpositions of these states, however, will change with time.

Figures 5 and 6 show the time evolution of a particularly interesting type of state for harmonic oscillator systems, a coherent state. This state is a superposition of an infinite number of two-particle eigenfunctions 
(a)
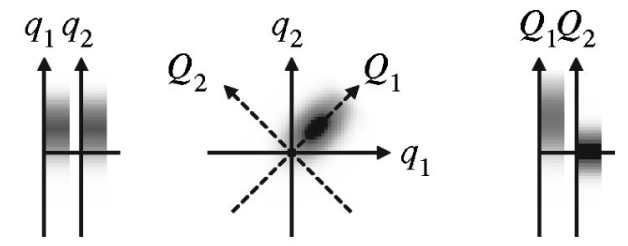

(b)
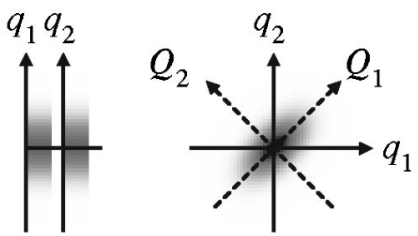

(c)
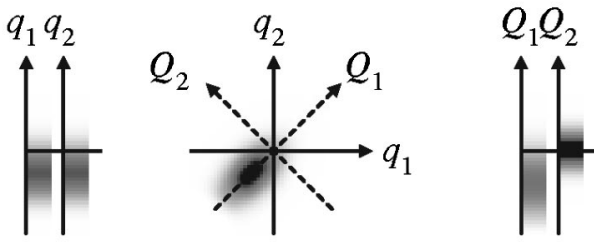

(d)
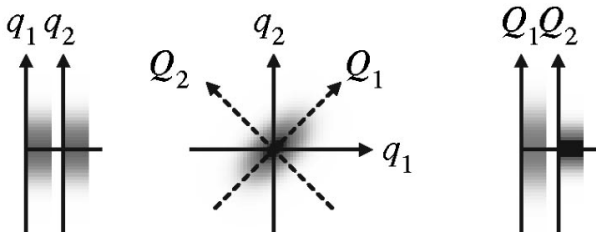

Fig. 5. Time evolution of a classical state of the two-particle system. In this state, the two masses are oscillating in synchronization in one of their eigenmodes.
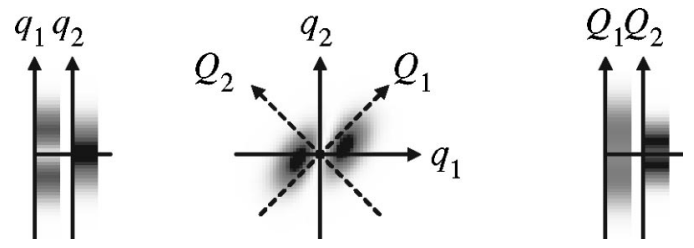

Fig. 7. Probablilty density for $\hat{q}_{1}^{\dagger}|0,0\rangle$.

$$
\begin{aligned}
\psi\left(Q_{1}, Q_{2}\right) & =\left\langle Q\left|\exp \left(-\frac{|\alpha|^{2}}{2}\right) \sum_{n=0}^{\infty} \frac{\alpha^{n}}{n !}\left(\hat{A}_{1}^{\dagger}\right)^{n}\right| 0,0\right\rangle \\
& =\exp \left(-\frac{|\alpha|^{2}}{2}\right) \sum_{n=0}^{\infty} \frac{\alpha^{n}}{\sqrt{n !}} \psi_{n}\left(Q_{1}\right) \psi_{0}\left(Q_{2}\right),
\end{aligned}
$$

where $\psi_{n}\left(Q_{1}\right)$ is the $n$th excited 1D harmonic oscillator wave function, evaluated along coordinate $Q_{1}$, and $\alpha$ is a constant specifying the amplitude of oscillation. (See Ref. 3 for other excellent illustrations of this type of state.) These states feature a single peak that is a Gaussian along any eigenmode axis and that stays Gaussian as it moves in an elliptical orbit around the origin. They are often called classical states because the center of the peak follows the trajectory of a classical particle.

This particularly strong correspondence between the time evolution of a quantum mechanical wave function and the motion of a classical particle makes coherent states particularly useful for demonstrating the transition from classical to quantum mechanical models of systems. For example, Fig. 5 shows a state that corresponds to the classical oscillators oscillating in synchronization, like Fig. 3(a). The $q_{1}$ and $q_{2}$ plots show the particles oscillating in synch with each other and the $Q_{1}$ and $Q_{2}$ plots show that only one eigenmode is excited. Figure 6 shows an oscillation corresponding to the other classical eigenmode where the two masses oscillate against each other, like Fig. 3(b). The $q_{1}$ and $q_{2}$ plots again show this behavior in an intuitive way.

Another interesting wave function is generated by the operator $\hat{q}_{1}$, which can be calculated from the coordinate transforms of Eq. (1). The resulting state $\hat{q}_{1}|0,0\rangle$ is shown in Fig. 7. This plot is similar to Fig. 4(c), which shows the state $\hat{Q}_{1}|0,0\rangle$. In particular, the $q_{1}-q_{2}$ plots for $\hat{q}_{1}|0,0\rangle$ look like the $Q_{1}-Q_{2}$ plots for $\hat{Q}_{1}|0,0\rangle$. There is a significant difference between these states, however. Because $\left\langle Q\left|\hat{Q}_{1}\right| 0,0\right\rangle$ is an eigenfunction of the system, it is constant in time, but $\left\langle Q\left|\hat{q}_{1}\right| 0,0\right\rangle$ is not an eigenfunction so it will change with time. Most notably, the two-peak pattern in the $q_{1}-q_{2}$ plots is not constant.

The main point of this section is to demonstrate in a visual way the requirements for plotting a system of two particles moving in one dimension. It requires a two-dimensional space to show the complete probability density, but some insight can be gained from groups of 1D projections. The two sets of axes that are most useful for projections are the position coordinate axes $q_{1}$ and $q_{2}$ and the normal mode coordinate axes $Q_{1}$ and $Q_{2}$.

\section{AN EIGHT-PARTICLE LATTICE}

The visualization techniques introduced in Sec. II can be extended to higher dimensions to show the lattice vibrations
Fig. 6. Time evolution of a classical state of the two-particle system, shown at a different time scale than Fig. 5. In this eigenmode, the two masses oscillate against each other. 


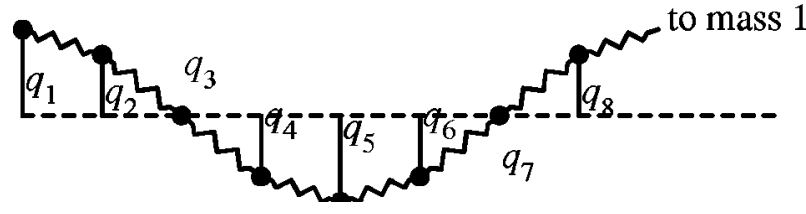

Fig. 8. The eight-particle system of harmonic oscillators connected by springs of spring constant $\kappa$. All masses are constrained to move vertically along the coordinates $q_{1}$ through $q_{8}$. Mass 8 is connected by a spring to mass 1 , as if the masses were arranged in a ring, giving periodic boundary conditions.

of a crystal (that is, phonons). As with the two-particle oscillator, we first consider the classical case. A system with eight oscillators is shown in Fig. 8. This system has periodic boundary conditions, which is a popular choice ${ }^{4-6}$ for demonstrating propagating waves. The masses and the spring constants are all identical. The position space coordinates are $q_{1}$ through $q_{8}$ and are collectively referred to as $q_{x}$ where the index $x$ ranges from 1 through 8 . The momenta of the masses, which are directed vertically along each axis, are $p_{1}$ through $p_{8}$ or collectively $p_{x}$ with $x$ ranging from 1 through 8 .

As with the two-particle problem, the key is to find the normal modes. The normal mode coordinates we will use are given by discrete cosine or sine transforms,

$$
\begin{aligned}
Q_{k} & =\sum_{x=1}^{8} \frac{1}{\sqrt{8}} q_{x} \cos \frac{\pi}{4} k x \quad(k=0,1,2,3,4), \\
Q_{k} & =-\sum_{x=1}^{8} \frac{1}{\sqrt{8}} q_{x} \sin \frac{\pi}{4} k x \quad(k=-1,-2,-3),
\end{aligned}
$$

and are collectively referred to as $Q_{k}$. A positive $k$ value indicates a "cosine mode" and a negative $k$ value indicates a "sine mode." Modes with identical $|k|$ are degenerate; they have identical energies and frequencies. For example, the cosine mode $Q_{1}$ is degenerate with the sine mode $Q_{-1}$. These normal mode coordinates are similar to, but distinct from, the more commonly used coordinates based on a discrete Fourier transform $Q_{F, k}=\sum_{x=1}^{8}(1 / \sqrt{8}) q_{x} e^{i \pi / 4 k x}$. The Fourier modes are linear combinations of the modes we use, $Q_{F, 1}=(1 / \sqrt{2})\left(Q_{1}+i Q_{-1}\right)$ and $Q_{F,-1}=(1 / \sqrt{2})\left(Q_{1}-i Q_{-1}\right)$. Conversely, our modes are linear combinations of the Fourier modes, $Q_{1}=1 / \sqrt{2}\left(Q_{F, 1}+Q_{F,-1}\right) \quad$ and $Q_{-1}=i / \sqrt{2}\left(Q_{F, 1}\right.$ $\left.-Q_{F,-1}\right)$. Fourier transform modes are complex, which makes them easier to manipulate than the pair of sine and cosine modes, but also makes them more difficult to plot. Because the goal of this article is to demonstrate phonon modes graphically, we have chosen the pure real sine and cosine modes.

The conjugate momenta (see the discussion on momenta below) for these normal mode coordinates have nearly identical transforms,

$$
\begin{aligned}
& P_{k}=\sum_{x=1}^{8} \frac{1}{\sqrt{8}} p_{x} \cos \frac{\pi}{4} k x \quad(k=0,1,2,3,4), \\
& P_{k}=\sum_{x=1}^{8} \frac{1}{\sqrt{8}} p_{x} \sin \frac{\pi}{4} k x \quad(k=-1,-2,-3),
\end{aligned}
$$
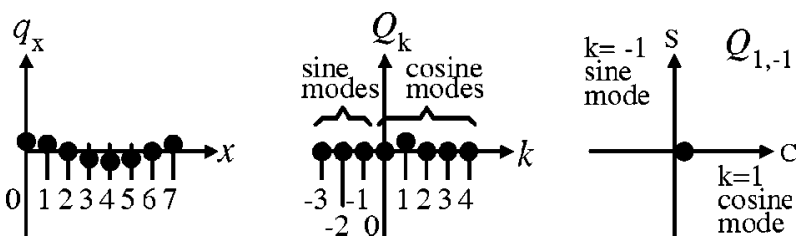

Fig. 9. The coordinate axes used to plot the eight-particle lattice.

and are collectively called $P_{k}$. The oscillation frequency for each normal mode is given by

$$
\omega_{k}=2 \sqrt{\frac{\kappa}{m}} \sin \frac{\pi}{2} \frac{|k|}{4},
$$

which is the dispersion relation for this system. As for the two-particle case, the reason for using the normal mode coordinates is that the equations of motion are decoupled using these coordinates.

The locations of all the masses, $q_{x}$, can be specified by a single point in an eight-dimensional space. Because such a space cannot be drawn clearly on a two-dimensional page, we draw only the projections onto various axes in this space. The axes that will be used in this section are shown in Fig. 9.

The position axes $q_{x}$ are on the left of each set of plots. The eight dots give the positions of the eight masses. Rather than draw and label each of the eight position axes, $q_{1}$ through $q_{8}$, we draw a single vertical axis labeled $q_{x}$ and label the horizontal axis the $x$ axis. The variable $x$ is a dimensionless index; the distance from the leftmost mass to mass number $x$ is $x \sigma$, where $\sigma$ is the distance between each mass.

The normal mode axes $Q_{k}$ are used in the center plot. As with the $q_{x}$ axes, a single vertical axis labeled $Q_{k}$ is drawn at $k=0$ and the horizontal axis is labeled $k$. The use of the letter $k$ intentionally suggests a momentum, however, $k$ is not the conjugate momentum. Like $x, k$ is a dimensionless index. The value of $k$ is the wave number of the mode, which for this system is the number of complete wave cycles in the lattice. (See the momentum discussion below.)

The $Q_{1,-1}$ plot shows the projection onto both $|k|=1$ axes simultaneously as a single point on a plane. The plot contains no new information; it is simply a different view of the two axes $Q_{1}$ and $Q_{-1}$ which are already shown in the $Q_{k}$ plot. However, it is useful because it shows more clearly the time behavior of these two degenerate modes.

Figure 10 shows all eight normal modes for this system. Each eigenmode has a particularly simple projection on the $Q_{k}$ axes. Most of the normal modes (except for $k=0$ and $k$ $=4)$ are degenerate pairs, with identical resonant frequencies and identical wavelengths on the position axes. One mode of each of these pairs corresponds to a sine function and the other to a cosine function. Linear combinations of these mode pairs can produce sinusoidal waves with varying phase angles. Figure 11 shows such a wave propagating in time. Note the time behavior of the projection on the $Q_{1,-1}$ plane - the projected point moves in a circle as the wave propagates along the position axes.

This system has two different momentum concepts, conjugate momentum and wave number, which can be difficult to grasp. ${ }^{7}$ The conjugate momentum is proportional to the time derivative of the coordinates. It is the familiar Newton- 
(a)<smiles>[Y]C(=C)C</smiles><smiles>CC(C)(C)[13CH]</smiles>

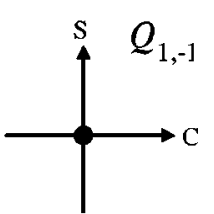

(b)
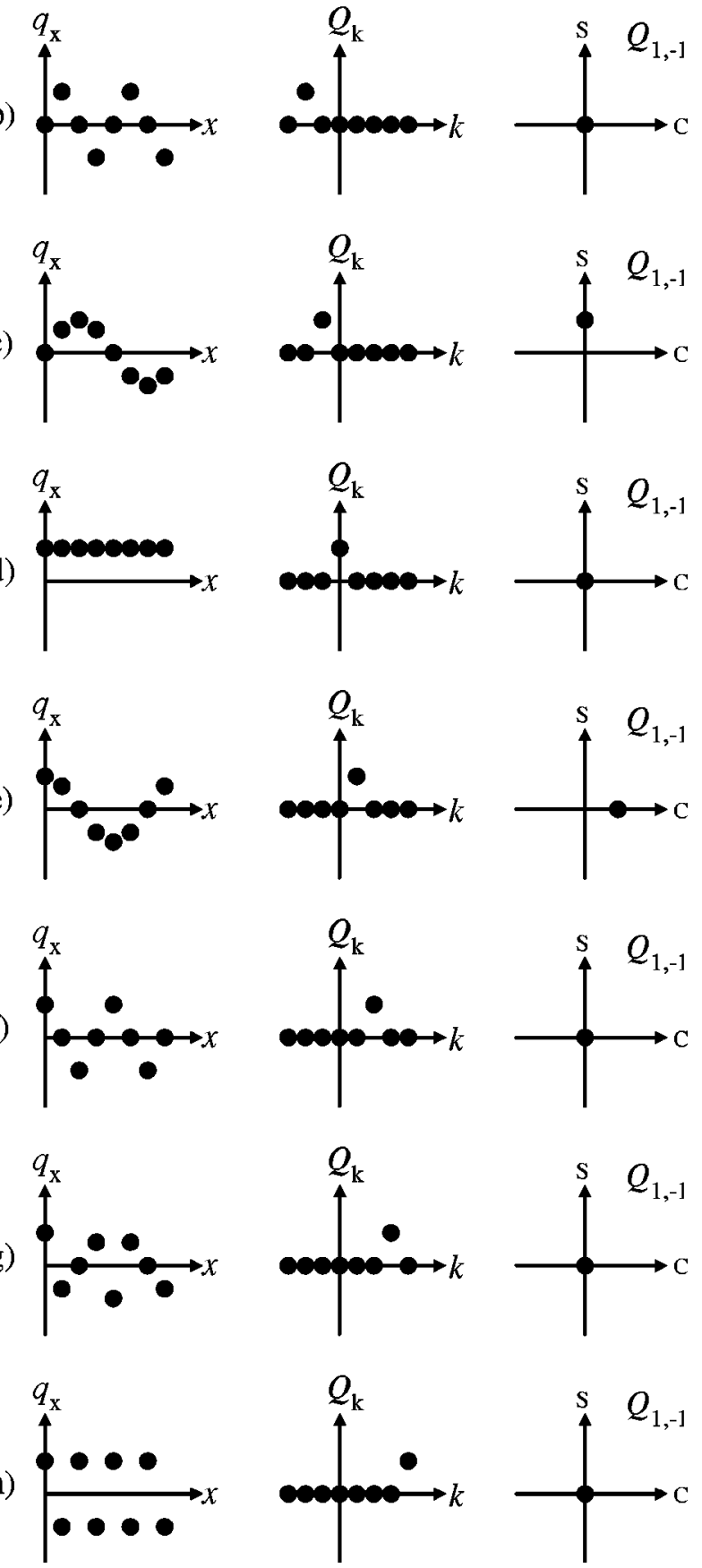

Fig. 10. All eight normal modes of the eight-particle lattice, from $k=-3$ in (a) to $k=4$ in (h), displayed using the coordinates used in Sec. III.

ian momentum of each of the masses in the chain. For this system, it is only directed vertically. Each of the eight individual masses has a time-varying value for this momentum. The other momentum concept, the wave number $k$, is a bit more subtle. It is also called the phonon momentum or the crystal momentum and is not a physical momentum. ${ }^{4}$ Instead, (a)
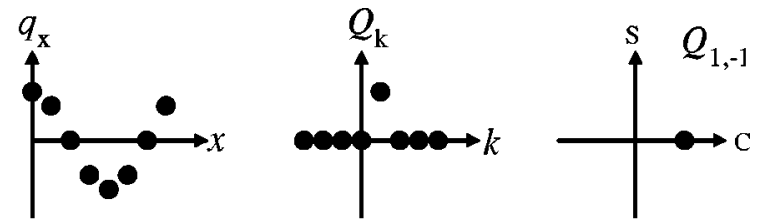

(b)
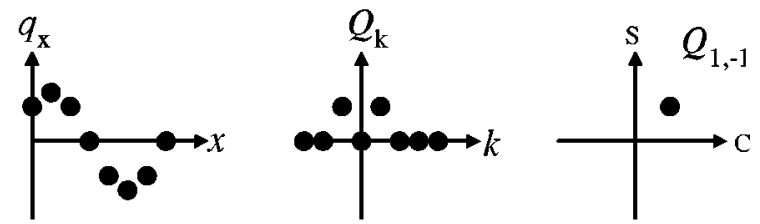

(c)
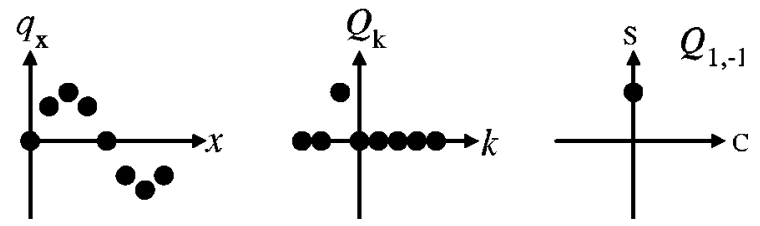

(d)
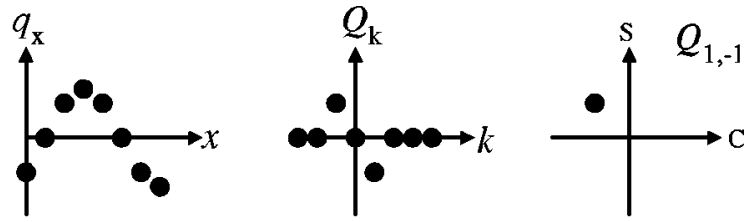

(e)
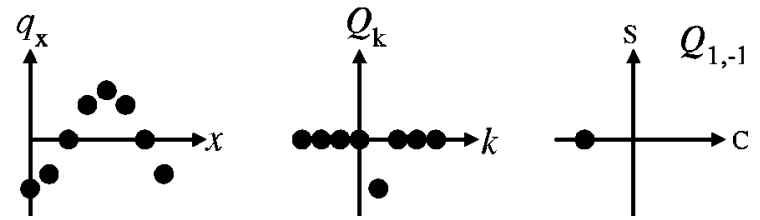

Fig. 11. Time evolution of the system showing a propagating $|k|=1$ wave.

it is a quantity that is used in conservation laws for interactions. The phonon momentum for this system is directed horizontally along the $x$ axis.

The following example qualitatively illustrates the nature of phonon momentum. Consider a model for the absorption of light by an ionic crystal. We neglect absorption by the electrons and assume all the light is absorbed as the ions vibrate in the electromagnetic field of the light (which is a good model for an ionic crystal absorbing infrared light). Absorption is proportional to the amplitude of vibration of the ions, so light will be appreciably absorbed only when the ions are vibrating near one of their resonant frequencies. For a lattice, the resonant frequencies are the normal mode frequencies of Eq. (16) and each corresponds to a particular normal mode with a particular wave number $k$. Light with both the right frequency $\omega_{k}$ and wave number $k$ will push all the ions of the crystal in the right direction at the right time to increase the amplitude of a particular normal mode. Light at a frequency $\omega_{k^{\prime}}$ but with a wave number $k^{\prime \prime}$ that does not match the wave number of the normal mode will push different parts of the crystal out of phase with each other and will not increase the amplitude of the normal mode. Thus, the light must have the same frequency and wave number as a particular phonon mode to interact with it. So, how is the phonon momentum a conserved quantity? As the light is absorbed, its amplitude at a particular $k$ decreases and the amplitude of the oscillation of the crystal at the same $k$ increases. In this sense, the quantity "amplitude at $k$ " is 
conserved. Quantum mechanically, the amplitudes are quantized and an interaction term in the Hamiltonian will be of the form $\hat{B}_{k} \hat{A}_{k}^{\dagger}$, where $\hat{B}_{k}$ removes a photon of wave vector $k$ from the electromagnetic field and $\hat{A}_{k}^{\dagger}$ adds a phonon of wave vector $k$ to the crystal. This is part of the fascinating topic of interacting quantum fields, which is beyond the scope of this article.

We next analyze this eight-particle system quantum mechanically. As with the two-particle system, we seek state vectors $|\psi(t)\rangle$ that satisfy the Schrödinger equation

$$
i \hbar \frac{d}{d t}|\psi(t)\rangle=\hat{H}|\psi(t)\rangle .
$$

In position coordinates, the Hamiltonian is

$$
\hat{H}=\sum_{x=1}^{8} \frac{\hat{p}_{x}^{2}}{2 m}+\sum_{x=1}^{8} \frac{1}{2} m \omega^{2}\left(\hat{q}_{x+1}-\hat{q}_{x}\right)^{2},
$$

which again does not give a separable wave equation because of the $\left(\hat{q}_{x+1}-\hat{q}_{x}\right)^{2}$ term. In normal mode coordinates

$$
\hat{H}=\sum_{k=-3}^{4} \frac{\hat{P}_{k}^{2}}{2 m}+\sum_{k=-3}^{4} \frac{1}{2} m \omega_{k}^{2} \hat{Q}_{k}^{2},
$$

which does give a separable wave function. The eightdimensional wave function can be written as a product of $1 \mathrm{D}$ harmonic oscillator wave functions,

$$
\begin{aligned}
\psi\left(Q_{-3}, Q_{-2}, \ldots, Q_{4}\right) & =\prod_{k=-3}^{4} \psi_{k}\left(Q_{k}\right) \\
& =\left\langle Q \mid n_{-3}, n_{-2}, n_{-1} ; n_{0}, n_{1}, n_{2}, n_{3}, n_{4}\right\rangle,
\end{aligned}
$$

where each $\psi_{k}$ can be any function of one variable. The Dirac-style notation on the last line is defined similarly to that of the two-particle system and is a particularly useful notation for the state of the system. The quantum mechanical state of such a many-particle system is often called a Fock state.

Projecting the eight-dimensional probability density function onto one of the normal modes is easy,

$$
P\left(Q_{1}\right)=\left|\psi_{1}\left(Q_{1}\right)\right|^{2},
$$

but it is not so easy to project it onto one of the $q_{x}$ axes,

$$
P\left(q_{1}\right)=\int_{-\infty}^{\infty} d q_{2} d q_{3} d q_{4} d q_{5} d q_{6} d q_{7} d q_{8}|\psi|^{2} .
$$

The integration over a seven-dimensional subspace of the eight-dimensional function must be done numerically. We use a Monte Carlo method as follows. ${ }^{8}$ We generate a large number (typically $10^{5}$ ) of random points in the eightdimensional $Q_{k}$ space with a distribution that matches the probability density in that space. This is done using the Metropolis algorithm. ${ }^{9}$ Each of those points is transformed to $q_{x}$ coordinates using the inverse transforms of Eq. (14). A histogram is constructed for each $q_{x}$ and these histograms are plotted on the $q_{x}$ vs $x$ plots, with greater numbers of points corresponding to darker shades of gray.

The ground state of the eight-particle quantum mechanical system is shown in Fig. 12 and is written as $|0,0,0 ; 0,0,0,0,0\rangle$ or simply $|0\rangle$. The projections onto the $q_{x}$ axes are Gaussian and are all identical. The projections onto
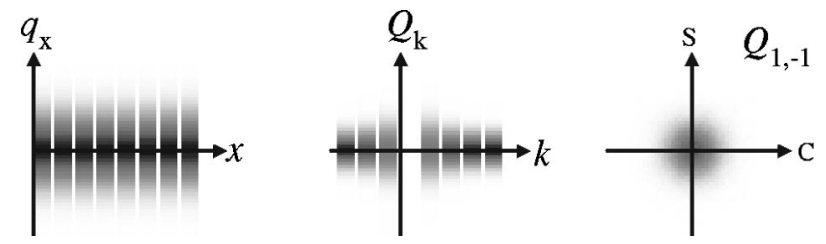

Fig. 12. Ground state of the quantum mechanical lattice. The apparent absence of a $Q_{0}$ projection is discussed in the text.

the $Q_{k}$ axes are also Gaussian with varying widths. Higher $|k|$ values have narrower Gaussian profiles, corresponding to the higher energies and higher frequencies of these modes, as specified in the dispersion relation of Eq. (16).

The $Q_{0}$ mode in Fig. 12 appears to be missing because we have chosen a delta function $\psi\left(Q_{0}\right)=\delta\left(Q_{0}\right)$ for the $Q_{0}$ normal mode. Thus, the ground state wave function is

$$
\langle Q \mid 0\rangle=\delta\left(Q_{0}\right) \prod_{k \neq 0} \psi_{0}\left(Q_{k}\right),
$$

where $\psi_{0}\left(Q_{k}\right)$ is the $1 \mathrm{D}$ ground state harmonic oscillator wave function for normal mode $k$. Normal mode $Q_{0}$ represents the motion of the center of mass of the entire system. Our eight-particle system is not anchored to any fixed reference system because of periodic boundary conditions, so the motion of its center of mass coordinate $Q_{0}$ is that of a free particle in space. Because the probability density for a free particle is uniform over space, using the free particle wave function for $\psi_{0}\left(Q_{0}\right)$ would produce a uniform probability density for all $q_{x}$ coordinates, which would not be useful for visualization. So, instead, we use the center of mass of the system as the origin for our coordinate system. This choice is equivalent to transforming into the center of mass reference frame. The result is a delta function for the probability density of $Q_{0}$.

As for the two-particle system normal modes, or indeed any $1 \mathrm{D}$ harmonic oscillator, we can define raising and lowering operators for this system. Each normal mode coordinate has one raising and one lowering operator,

$$
\begin{aligned}
& \hat{A}_{k}^{\dagger}=\sqrt{\frac{m \omega_{k}}{2 \hbar}} \hat{Q}_{k}-i \sqrt{\frac{1}{2 m \omega_{k} \hbar}} \hat{P}_{k}, \\
& \hat{A}_{k}=\sqrt{\frac{m \omega_{k}}{2 \hbar}} \hat{Q}_{k}+i \sqrt{\frac{1}{2 m \omega_{k} \hbar}} \hat{P}_{k} .
\end{aligned}
$$

The action of these operators on a Fock space ket is to raise or lower one of the $n_{k}$ values, the energy eigenstate of the corresponding mode. For example, the action of the $k=1$ raising operator is $\hat{A}_{1}^{\dagger}\left|n_{-3}, n_{-2}, n_{-1} ; n_{0}, n_{1}, n_{2}, n_{3}, n_{4}\right\rangle$ $=\sqrt{n_{1}+1}\left|n_{-3}, n_{-2}, n_{-1} ; n_{0}, n_{1}+1, n_{2}, n_{3}, n_{4}\right\rangle$ and of the $k$ $=1$ lowering operator is $\hat{A}_{1} \mid n_{-3}, n_{-2}, n_{-1} ; n_{0}, n_{1}, n_{2}, n_{3}$,
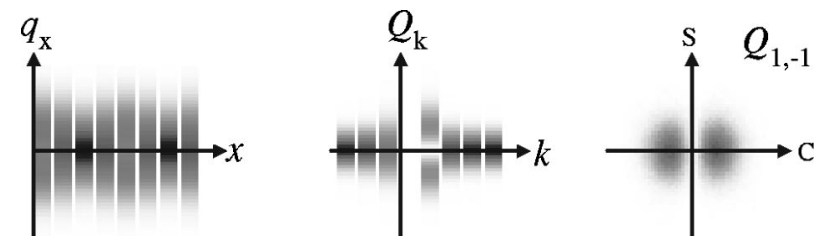

Fig. 13. The quantum mechanical lattice with one $k=1$ phonon, $\hat{A}_{1}^{\dagger}|0\rangle$. 

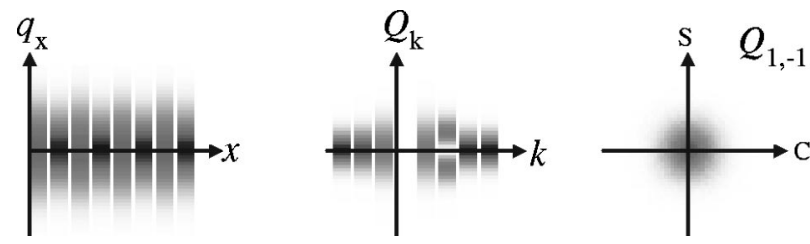

Fig. 14. The quantum mechanical lattice with one $k=2$ phonon, $\hat{A}_{2}^{\dagger}|0\rangle$.

$\left.n_{4}\right\rangle=\sqrt{n_{1}}\left|n_{-3}, n_{-2}, n_{-1} ; n_{0}, n_{1}-1, n_{2}, n_{3}, n_{4}\right\rangle$. Note that the action of the $k=1$ lowering operator is not the same as that of the $k=-1$ raising operator, which is $\hat{A}_{-1}^{\dagger} \mid n_{-3}, n_{-2}$, $\left.n_{-1} ; n_{0}, n_{1}, n_{2}, n_{3}, n_{4}\right\rangle=\sqrt{n_{-1}+1} \mid n_{-3}, n_{-2}, n_{-1}+1 ; n_{0}, n_{1}$, $\left.n_{2}, n_{3}, n_{4}\right\rangle$.

The word phonon refers to an increase in the excitation number of the system. The $\hat{A}_{k}^{\dagger}$ operators add one phonon each and the $\hat{A}_{k}$ operators remove one phonon each from a system. The ground state has zero phonons. Using an $\hat{A}_{k}^{\dagger}$ operator on the ground state creates a one-phonon state. For example, $\hat{A}_{1}^{\dagger}|0,0,0 ; 0,0,0,0,0\rangle=|0,0,0 ; 0,1,0,0,0\rangle$ is a system with one $k=1$ phonon. A more compact notation is $\hat{A}_{1}^{\dagger}|0\rangle$. This one-phonon state is shown in Fig. 13. In the normal mode space, we see that the probability along $Q_{1}$ is that of a first excited harmonic oscillator and all the other normal modes are in their ground states. The $Q_{1,-1}$ axes emphasize that the first excited state is along a cosine mode. On the $q_{x}$ position axes, we do not see a cosine wave as we might expect. The expectation value of the position for any mass is still zero. However, the width of the probability distribution now varies with $x$ and this variation follows a cosine function.

Similar observations apply to Fig. 14, which shows the result of $\hat{A}_{2}^{\dagger}|0,0,0 ; 0,0,0,0,0\rangle=|0,0,0 ; 0,0,1,0,0\rangle$ or $\hat{A}_{2}^{\dagger}|0\rangle$. This system has one $k=2$ phonon. Its $Q_{2}$ probability density is that of a first excited harmonic oscillator state. The width of its $q_{x}$ probability densities vary with $x$ following a cosine function, except that the wavelength of this cosine function is shorter than for the $k=1$ phonon.

We can put several phonons into a system by using the raising operator several times. For example, $\left(\hat{A}_{1}^{\dagger}\right)^{4}|0\rangle$ $=|0,0,0 ; 0,4,0,0,0\rangle$ is a system with four $k=1$ phonons and is shown in Fig. 15. The $Q_{k}$ plot shows that the $k=1$ normal mode is in its fourth excited state and the $q_{x}$ plot clearly shows the cosine variation in the width of the probability density of the masses. The amplitude of the width variation is greater with more phonons in the system.

A system can also contain several phonons of arbitrary $k$ values. Figure 16 shows the system with three $k=1$ phonons and one $k=-1$ phonon. This system has the same number of
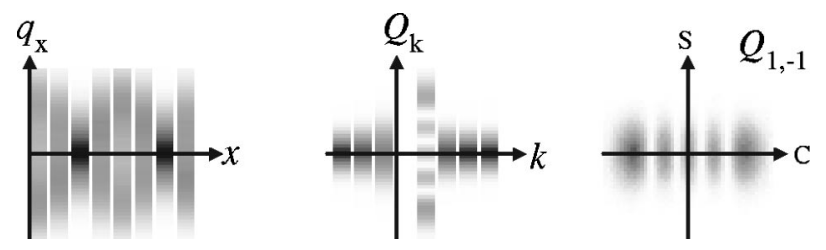

Fig. 15. The quantum mechanical lattice with four $k=1$ phonons, $\left(\hat{A}_{1}^{\dagger}\right)^{4}|0\rangle$.
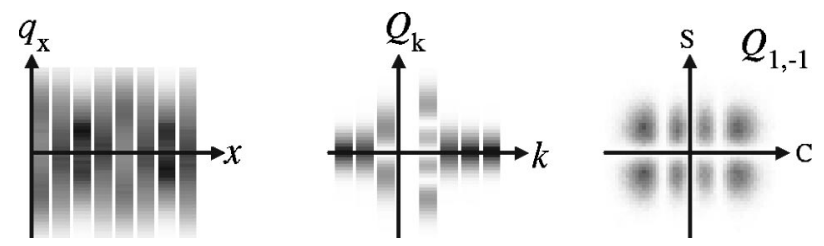

Fig. 16. The quantum mechanical lattice with four $k=1$ phonons, three in cosine modes and one in a sine mode, $\left(\hat{A}_{1}^{\dagger}\right)^{3}\left(\hat{A}_{-1}^{\dagger}\right)^{1}|0\rangle$.

phonons as the one in Fig. 15, and because they are all $|k|$ $=1$ phonons, the total energy of both systems is identical. The $q_{x}$ projection still shows an expectation value of zero for each mass.

To get a nonzero position expectation value for any of the masses, a superposition of states is required. Figure 17 shows a superposition of the ground state and a one-phonon state. The probability densities of many of the masses are now clearly centered above or below the equilibrium position, indicating a nonzero expectation value. Figure 17 shows the time evolution of this state by showing it at three separate times. The peak of $q_{x}$ probabilities follows a cosine shape in space and oscillates in time like a vibrating string.

The time behavior of these states is calculated from the Schrödinger equation. Each 1D harmonic oscillator wave function is multiplied by a phase factor $e^{i \omega_{k} t}$, where $\omega_{k}$ is determined by the dispersion relation of Eq. (16). The norm of the resulting wave function gives the probability density that is plotted.

At this point, we have introduced all the background needed to understand the most important plot in this paper, Fig. 18. It is a coherent state similar to those introduced in the discussion of the two-particle system. To get a coherent state in any one mode, we would use an expression similar to Eq. (13),

$$
\exp \left(-\frac{|\alpha|^{2}}{2}\right) \sum_{n=0}^{\infty} \frac{\alpha^{n}}{n !} e^{i \phi_{n}}\left(\hat{A}_{k}^{\dagger}\right)^{n}|0\rangle
$$

(a)

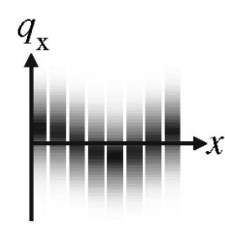

(b)

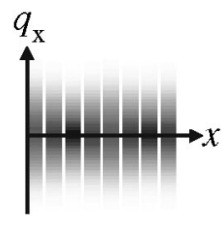

(c)

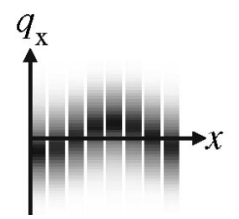

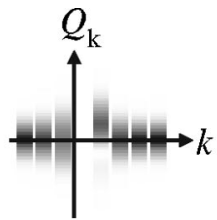
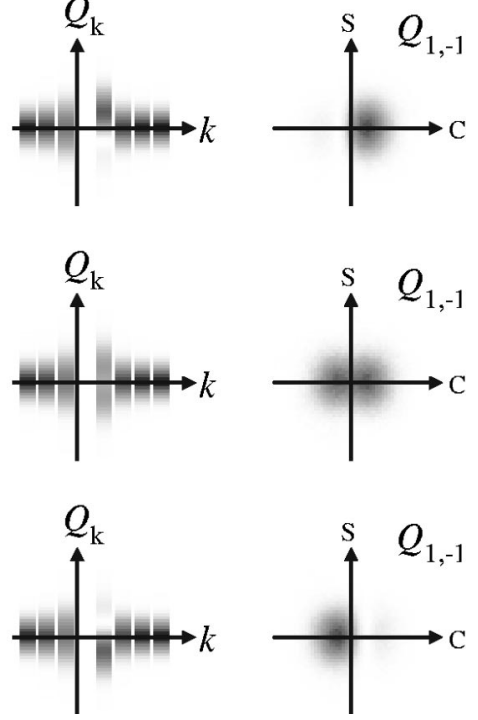

Fig. 17. Time sequence for the quantum mechanical lattice in a superposition of states, the ground state and a one-phonon state, $1 / \sqrt{2}\left[\hat{A}_{1}^{\dagger}|0\rangle+|0\rangle\right]$. 
(a)
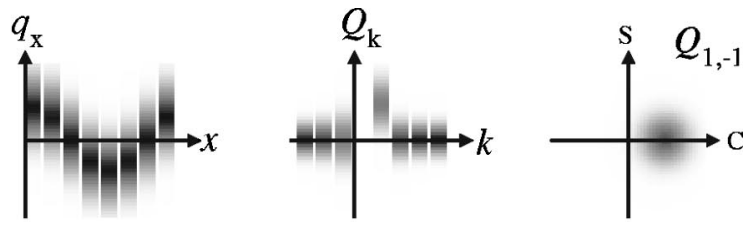

(b)
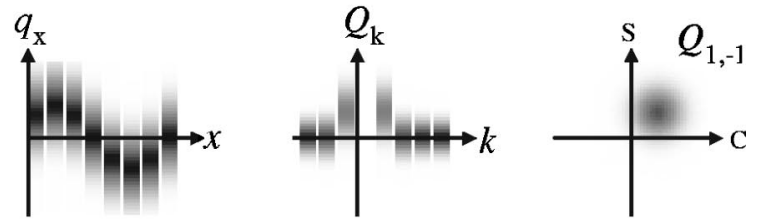

(c)
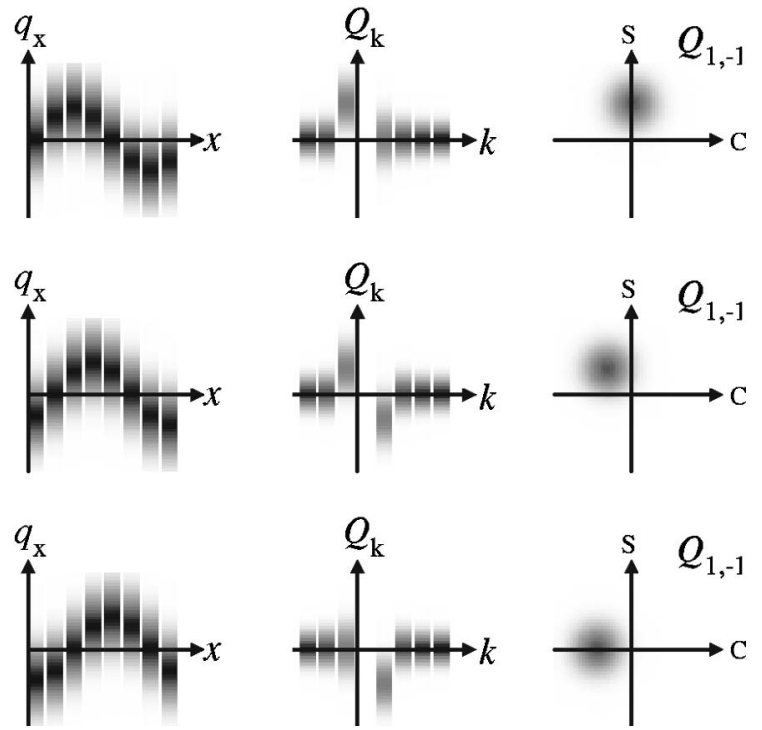

Fig. 18. Time evolution of the quantum mechanical lattice in a coherent, propagating $|k|=1$ state. This is a good visualization of a sound wave in a solid.

where $\phi_{n}=\left(n+\frac{1}{2}\right) \omega_{k} t$ gives the time dependence. This state would produce a coherent standing wave corresponding to mode $k$. However, Fig. 18 is a propagating wave which requires a superposition of a cosine mode and a sine mode with the right relative phase. Such a state is constructed by

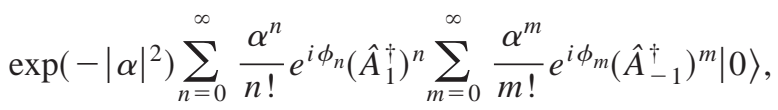

with $\phi_{n}=\left(n+\frac{1}{2}\right) \omega_{1} t$ and $\phi_{m}=\left(m+\frac{1}{2}\right)\left(\omega_{-1} t-\pi / 2\right)$, showing that the sine modes are shifted by $-\pi / 2$ relative to the cosine modes. (With no shift, the result would be another standing wave.) Figure 18 shows the time evolution of such a $|k|=1$ propagating coherent state.

The $q_{x}$ projection is the most pedagogically useful. If we compare Fig. 18 with the classical propagating wave in Fig. 11 , we see that the pictures look qualitatively similar with a sinusoidal wave propagating to the right. In the quantum case, however, the locations of each mass are not certain, but are given by a probability distribution that is centered on the classical location. We claim that Fig. 18 is an intuitive and correct picture of a propagating phonon. It is important to note that this picture is of a rather special, coherent state instead of a single phonon. Still, the idea of replacing the definite classical values of the positions of each mass with a
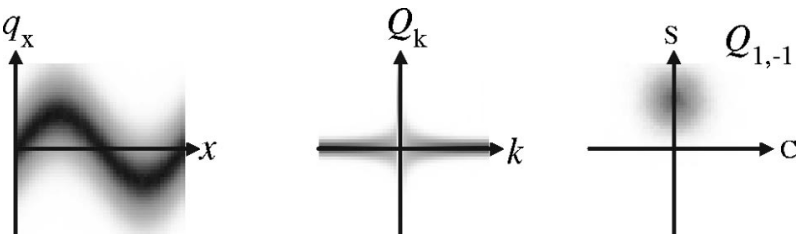

Fig. 19. A fifty-particle phonon system in a coherent $k=-1$ (sine-type mode) state.

probability distribution is true for all states of this system, and the coherent state simply makes the connection to the classical system most vivid.

In fact, the basic idea of replacing each classical value with a probability distribution applies to any quantum field, and we believe that this idea is a good way to introduce quantum field theory. Figure 19 shows a coherent state for a fifty-particle quantum mechanical lattice. Using fifty particles makes this system a good approximation to a continuous, scalar field in one dimension.

The coherent propagating waves of Figs. 18 and 19 are good approximations to several real-world quantum fields. For a lattice, these represent sound waves traveling through the lattice. In contrast, noncoherent sums of phonon states (in the proper proportions) can represent thermal vibrations of the lattice. Using the scalar field to represent one component of an electromagnetic field, Figs. 18 and 19 are qualitatively correct representations of the coherent light of a laser. They could also represent the electric waves broadcast by a radio antenna. In contrast, noncoherent sums of these electric field states can represent thermal radiation.

It is interesting to see a few other states of the eightparticle system. Figure 20 shows a noncoherent sum of several $|k|=1$ states, each with the same energy. Without coherence, though, the $q_{x}$ probability distributions are all centered on zero. This sum of states is a good analogy for monochromatic but noncoherent light.

Figure 21 shows a squeezed state. ${ }^{10}$ Squeezed states of light are similar to laser light and can be produced by various nonlinear optical techniques. A squeezed state is similar to a coherent state, but the width of the Gaussian function plotted in the $Q_{1,-1}$ plane varies with time. The squeezing is greatest in Fig. 21(c), where the width of the Gaussian is a minimum. A measurement of the $|k|=1$ modes of the system at that time will have less uncertainty than a measurement on an otherwise identical coherent state. This squeezing is balanced by the width of the Gaussian in Figs. 21(a) and 21(e), which is larger than for an otherwise identical coherent state. Thus, a squeezed state allows the system to have a lower uncertainty in its position at some times, but compensates by having a higher uncertainty at other times. This reduction in uncertainty can be used to improve the sensitivity of some types of measurements, for example, a gravitational wave detector. ${ }^{11}$
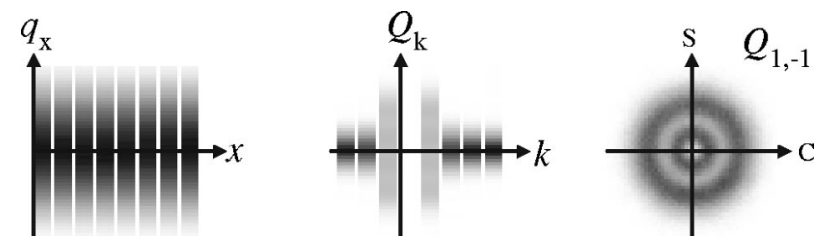

Fig. 20. A noncoherent superposition of several $|k|=1$ four-phonon states. 
(a)
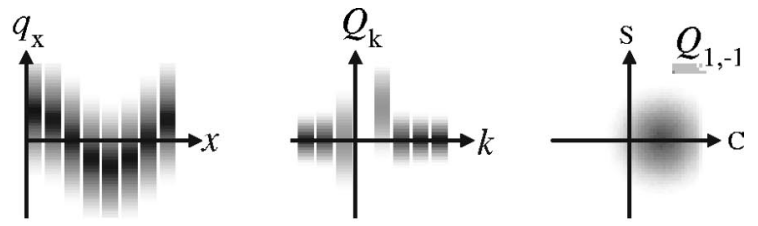

(b)
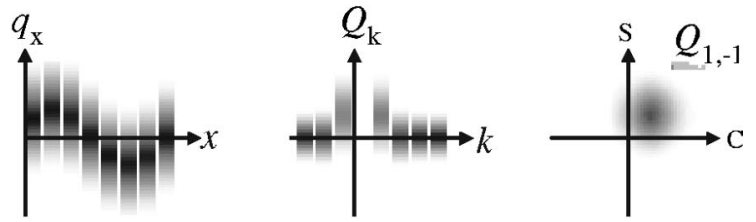

(c)
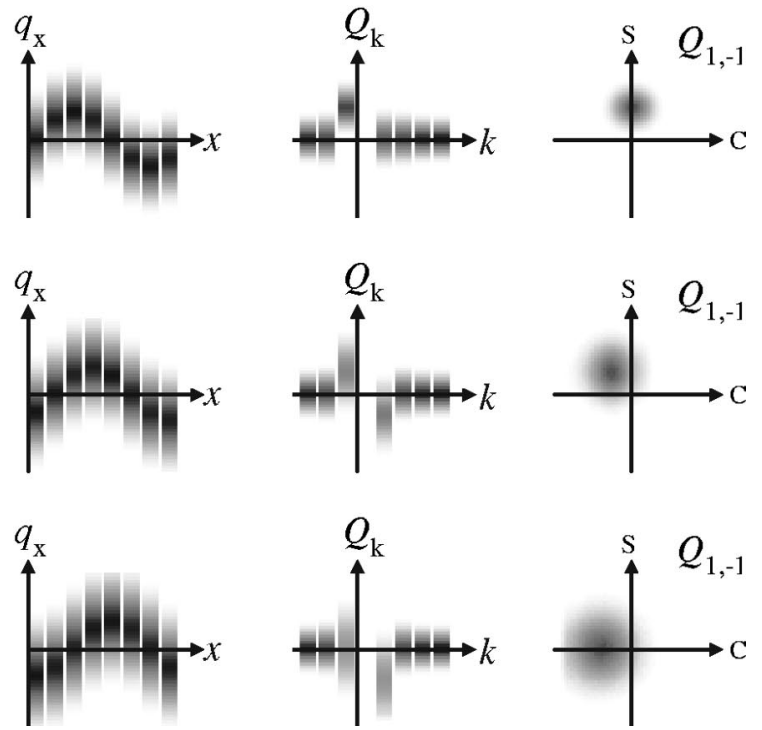

Fig. 21. Time development of a squeezed state.

Figure 22 shows a one-phonon state in one of the alternate discrete Fourier transform normal mode coordinates, discussed after Eq. (14). This state is a complex linear combination of the $k=1$ and $k=-1$ normal mode coordinates. It has the advantage that this one-phonon state represents a traveling wave. States with positive $k$ travel in one direction and negative $k$ travel in the other direction. However, a plot reveals why we chose not to use these coordinates - they are featureless on a probability density plot. If phase were plotted on these plots, such as by using a color code, a point of constant phase on the $Q_{ \pm 1}$ plot would travel in a circle at frequency $\omega_{k}$. (For beautiful pictures of one- and twodimensional wave functions showing phase information, see Ref. 12.)

It is possible, and often useful, to define an operator that creates a localized excitation of the lattice (for phonons) or the field (for photons and electrons and such). For our eight-
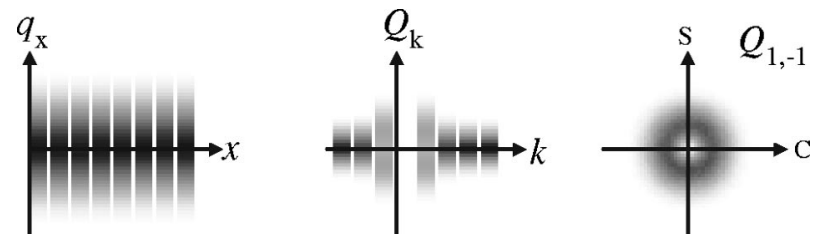

Fig. 22. A one-phonon state using one alternate normal mode coordinate, $\left[\hat{A}_{1}^{\dagger}+i \hat{A}_{-1}^{\dagger}\right]|0\rangle$.
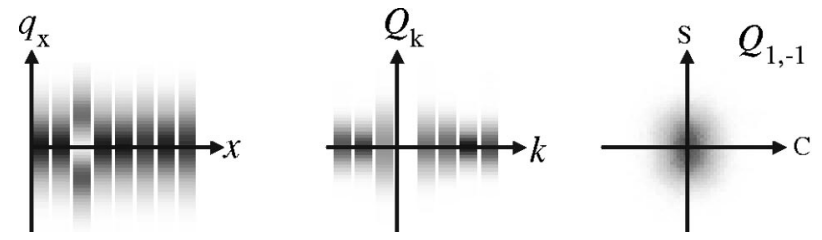

Fig. 23. A local excitation at $x=3$ from the $\hat{q}_{3}^{\dagger}$ field operator, $\hat{q}_{3}^{\dagger}|0\rangle$.

particle system, this is done by using the $\hat{q}_{x}$ operator, which is often called a field operator. Like $\hat{q}_{1}$ for the two-particle system, this operator can be expressed in terms of the normal mode operators using the inverse of the discrete sine and cosine transforms of Eq. (14),

$$
\hat{q}_{x}=\sum_{k=0}^{4} \frac{1}{\sqrt{8}} \hat{Q}_{k} \cos \frac{\pi}{4} k x+\sum_{k=-3}^{-1} \frac{1}{\sqrt{8}} \hat{Q}_{k} \sin \frac{\pi}{4} k x .
$$

Figure 23 shows the state $\hat{q}_{3}^{\dagger}|0\rangle$ which puts such a localized excitation at location $x=3$. The $q_{x}$ plot of this system is quite similar to the $Q_{k}$ plot of Fig. 13. In both plots, most of the coordinates have ground state probability densities and just one coordinate has a first excited state probability density. However, the $Q_{k}$ plot shows an eigenfunction so it will be stationary in time, whereas the $q_{x}$ plot shows a superposition so it will vary in time. In particular, the $q_{x}$ plot's appearance is short-lived and quickly decays to be barely distinguishable from the ground state.

The word "particle" has a different meaning in quantum field theory than it does in many other contexts. Usually, particle refers to an object localized in space. However, a phonon is usually called a particle, and it is distributed over the entire lattice. The same observation can be made, for example, for a photon (an excitation of the electromagnetic field) or for an electron (an excitation of its associated field, often called the Dirac field). The local excitation $\hat{q}_{x}^{\dagger}|0\rangle$ described above is not a phonon; it is in fact a linear combination of many phonon states. In some contexts, this local excitation is called a particle.

\section{CONCLUSION}

A one-dimensional lattice of $N$ coupled harmonic oscillators is a good demonstration system for phonons and, as $N$ $\rightarrow \infty$, for quantum fields. Classically, this system demonstrates the normal mode decomposition needed to solve such many-particle or field theory problems and also shows the two types of momentum, $p_{x}$ and $k$, that such systems have. Quantum mechanically, the wave function is a complex function of $N$ dimensions which cannot be plotted for $N>3$. However, the probability density is a positive, real number at each point in this $N$-dimensional space and can be projected onto groups of coordinate axes such as the $q_{x}$ position axes and $Q_{k}$ normal mode axes. The resulting probability density plots present a somewhat intuitive picture of the phonon wave function, or of the wave function of any quantum field. What they show for a particular state of the system, the coherent state, is that displacement of each classical mass is replaced by a probability density centered on the classical location. Thus, just like the location of a single particle is blurred in quantum mechanics, the location of every particle in a lattice, or every value of a field, is blurred in quantum 
field theory. We hope that these pictures can serve as an enticing introduction to the fascinating but difficult subject of quantum field theory.

\section{PROBLEMS}

(1) Find the time dependence of the positions of the two classical masses for each configuration in Fig. 3. Use $Q_{1 i}$ and $Q_{2 i}$ or $q_{1 i}$ and $q_{2 i}$ as the coordinates at time $t=0$. First use the $Q$ coordinates and then transform to the $q$ coordinates.

(2) Write explicit expressions for the four wave functions shown in Fig. 4.

(3) Show that $\hat{Q}_{1}|0\rangle$ is an eigenstate of the two-particle system, but that $\hat{q}_{1}|0\rangle$ is not. Hint: find $\hat{Q}_{1}$ and $\hat{q}_{1}$ in terms of $\hat{A}_{1}$ and $\hat{A}_{1}^{\dagger}$ using Eqs. (11) and (1) and show that operating on $|0\rangle$ with $\hat{Q}_{1}$ gives a single particle state, but that $\hat{q}_{1}$ gives a superposition of states.

(4) Find the time dependence of the positions of the eight classical masses in Fig. 11. Use $Q_{x i}$ or $q_{x i}$ as the coordinates at time $t=0$. First use the $Q$ coordinates, and then transform into the $q$ coordinates.

(5) (Advanced) Evaluate the expectation value for $\hat{Q}_{1}$ and $\hat{Q}_{2}$ for the states in Figs. 13 and 17 (at time $t=0$ only). Hint: the expectation value of $\hat{Q}_{k}$ for a state $|\psi\rangle$ is $\left\langle\psi\left|\hat{Q}_{k}\right| \psi\right\rangle$, where it is usually helpful to express $\hat{Q}_{k}$ and $|\psi\rangle$ in terms of $\hat{A}_{k}$ and $\hat{A}_{k}^{\dagger}$. For example, $|\psi\rangle=\hat{A}_{1}^{\dagger}|0\rangle$ and $\langle\psi|=\langle 0| \hat{A}_{1}$. Use the result that $\left\langle n_{-3} \ldots n_{4} \mid m_{-3} \ldots m_{4}\right\rangle=\prod_{k=-3}^{4} \delta_{n_{k} m_{k}}$. That is, $\left\langle n_{-3} \ldots n_{4} \mid m_{-3} \ldots m_{4}\right\rangle$ is 0 unless both sides have the same number in each mode. For more of this sort of problem, see Ref. 13. a) Present address: Intel Corporation, 5200 NE Elam Young Parkway, Mail Stop RA1-329, Hillsboro, Oregon 97124; electronic mail: sjohnson@landau.ucdavis.edu

b)Electronic mail: tgutierr@altair.ucdavis.edu

${ }^{1}$ The software, BlochApp, FieldApp, JCApp, and QEDApp, by G. Andrew Antonelli and Wolfgang Christian, is described in Denis Donnelly, "CIP's sixth annual software contest," Comput. Phys. 9, 594-601 (1995).

${ }^{2}$ Ramamurti Shankar, Principles of Quantum Mechanics (Plenum, New York, 1994), 2nd ed. Example 1.8.6 is the classical coupled harmonic oscillator, examples 10.1.3 and 10.2.2 treat the quantum version, and Chapter 7 is on the one-dimensional oscillator.

${ }^{3}$ Siegmund Brandt and Hans Dieter Dahmen, The Picture Book of Quantum Mechanics (Wiley, New York, 1985), Secs. 7.1, 7.2, 9.1, 12.3.

${ }^{4}$ Charles Kittel, Introduction to Solid State Physics (Wiley, New York, 1976), 6th ed., Chap. 4.

${ }^{5}$ Neil Ashcroft and N. David Mermin, Solid State Physics (Saunders College Publishing, 1976), p. 430.

${ }^{6}$ Walter Greiner and Joachim Reinhardt, Field Quantization (SpringerVerlag, Berlin, 1996), Chap. 1.

${ }^{7}$ John Robinson Pierce, Almost All About Waves (MIT, Cambridge, 1974), Chaps. 10 and 11.

${ }^{8}$ Verissimo M. de Aquino, Valdir C. Aguilera-Navarro, Mario Goto, and Hiromi Iwamoto, "Monte Carlo image representation," Am. J. Phys. 69, 788-792 (2001).

${ }^{9}$ See, for example, B. L. Hammond, W. A. Lester, Jr., and P. J. Reynolds, Monte Carlo Methods in Ab initio Quantum Chemistry (World Scientific, Singapore, 1994).

${ }^{10}$ Marlan O. Scully and M. Suhail Zubairy, Quantum Optics (Cambridge U.P., Cambridge, 1997).

${ }^{11}$ Peter Knight, "Quantum optics," in The New Physics, edited by Paul C. W. Davies (Cambridge U.P., Cambridge, 1992), Secs. 10.6 and 10.9.

${ }^{12}$ Bernd Thaller, Visual Quantum Mechanics: Selected Topics with Computer-Generated Animations of Quantum-Mechanical Phenomena (Springer/TELOS, Berlin, 2000).

${ }^{13}$ Rubin H. Landau, Quantum Mechanics II: A Second Course in Quantum Theory (Wiley, New York, 1996), Sec. 21.2, Coherent States of the Radiation Field: Tutorial. 